\title{
Aporia in the Maps of the Hypermedia Systems
}

\author{
Francisco Cipolla-Ficarra \\ HCI Lab - F\&F Multimedia Communic@ tions Corp., \\ Alaipo - Asociación Latina de Interacción Persona - Ordenador, and \\ Ainci - Asociación Internacional de Comunicación Interactiva \\ Via Pascoli, S. 15, C.P. 7 - 24121 Bergamo, Italy \\ ficarra@alaipo.com
}

\begin{abstract}
Aporia is a Greek word meaning helplessness or difficulty in dealing with, or finding out about, something. This investigation is aimed at determining the aporia existing in the present maps with satellite and traditional pictures. Moreover, we present a quality metric called antinomy, to eradicate the aporia in the design of interactive systems with cartographic contents. The present quality metrics can be applied to the digital maps. The importance of traditional iconography is also stressed in the maps and the need to carry out heuristic assessments before using a new iconography. We need to increase the quality in hypermedia systems, communicability and efficiency of the digital maps, quite aside from location, the multimedia technological support used in the presentation of the cartographic information into computers, palms, mobile phones, etc., and potential users of the interactive systems.
\end{abstract}

Keywords: Antinomy, Aporia, Cartography, Design, Evaluation, HCI, Hypermedia, Maps, Metrics, Quality, Semiotic.

\section{Introduction}

On many occasions researchers of semiotics have rejected the dichotomy between connotation and denotation because there are difficulties in reaching a clear distinction between the primary and secondary aspects of the meaning. For example, Roland Barthes usually speaks of denotation of the sign but not of connotation [1]. Umberto Eco retains the basic dichotomy, but since his theory of meaning rejects the dimension of reference, his concepts of denotation and connotation cover much of the traditional sphere of connotation. Denotation, according to Eco, is a "cultural unit, ... culturally recognized property of a possible referent" [2]. A connotation is also "a cultural unit" but it is "conveyed by its denotation and not necessarily corresponding to a culturally recognized property of the possible referent" [1]. Elsewhere, Eco defines a connotation as the "set of all cultural units which are institutionally associated in the receiver's mind with the signifier" [2]. The antinomy allows to establish a relation between primary and secondary meaning in a univocal way, for example: smoke and fire. However, it is necessary to establish a difference between natural and artificial signs. It is through semiotic convention that the sign exists, as long as a human group decides to use a thing as the vehicle of any other thing [2]. In 
cartography the use of the sky blue colour to indicate water and green to indicate areas of vegetation is a centuries-old convention, and this can easily be checked in any atlas printed on paper since the 15th century. The use of the current artificial signs in the hypermedia systems does not respect any kind of convention. In cartography the use of the sky blue colour to indicate water and green to indicate areas of vegetation is a centuries-old convention, and this can easily be checked in any atlas printed on paper since the 15th century. The use of the current artificial signs in the hypermedia systems does not respect any kind of convention. A classic example are the traffic signs for the viability of vehicles on streets, roads, highways, etc. which in many cases do not maintain that unique relationship in the same country. Consequently, to extrapolate these sign systems at world scale without previously carrying out an analysis, can entail negative consequences in the quality of the interactive cartographic systems.

The relationship between the icon and its meaning, strengthened by the text, does not always allow the elimination of the process of unlimited semiosis [1], which damages antinomy. In the interface design of hypermedia systems, some designers plump for guidelines in relation to the hardware and software that they use, i.e. Macintosh [3]. Others, in contrast, start to make icons taking as a reference the cultural environment where they are involved [4]. However, in both cases antinomy can be absent, because the problem of emulation and simulation of reality on the screens of the interactive systems, persists.

\section{Maps: Evolution of the Emulation and Simulation of Reality}

Currently cartography has reached a peak thanks to the new technologies, peak which is to be found in the intersection among of physical, social and digital domains. For example, in the human-computer interaction community explores the use of pervasive and/or ubiquitous computing technologies in inhabited environments [5], simulations for urban planning [6], helpful environment [7], designing effective digital systems inspired by, or included in, the physical world [8], etc. One of the classical problems in the evolution of maps has been the transition from spherical representation to plane representation. Once the main scales in cartography were determined, the next step was to analyze the different geometrical properties of the transformations. In the history of the evolution of the representation of maps we find three basic ways to depict planet Earth; cylindrical, conic and planar. Some examples of these models are: Lambert, 1772 (conic); Mercartor, 1569 (cylindrical), and Stereographic, Hipparchus, 160-125 B.C. (planar) [9]. Obviously, the Mercator map is an excellent example of map design for navigation purposes. This map replaced the previous model, known as gnomonic. Besides, Mercator introduced in his map, parallels and later on, meridians, which with the passing of the centuries helped to bring about a rectangular depiction of the globe. Several models and shapes were created throughout time. Some examples are: Sinusoidal (Cossin, 1570); Gall (Ortographic, 1855), and Robinson is the author of the ortophanic model (1963) -designed to look correct [9]. Only in the last half of the century has the map become more than a repository for information. Computerized cartography and modern photographic techniques have increased the density of information some 5,000-fold in the best of today's data maps compared to Halley's pioneering effort [10]. 
As we can see, a map is a schematic representation of reality that has evolved with the passing of time. It is not a natural language. It is a representation that has to be learned. The observer has to collect three abilities: the sight from above, the sense of direction and memory associated to $2 \mathrm{D}$ plan. The space-visual sense of direction requires a learning process in which the interactive systems can help, but we have to remember that feedback time must be immediate, i.e., a fraction of a second. Nevertheless, nowadays a certain slowness predominates in the moment of interaction with the image (Google Maps, TuttoCittà, ViaMichellin, etc.), even if the access and localization of the information required in the hyperbase management system is quick [11]. With the regard to the design of $3 \mathrm{D}$, it has a widespread diffusion in the lines of that presented to youngsters and inexperienced users at the Simcity [12]. The success of interactive games lies in their diachronic evolution, which has developed from a cartography for the cities in 2D, to 3D as years have gone by. Besides, it enables the user to regulate the angle of vision. We introduce the notion of "bird vision" in quality measurement into interactive cartography. This belongs to the category of presentation and it refers to the angle of vision of the observer that is regarded as ideal for the correct fruition of the maps, $2 \mathrm{D}$ and $3 \mathrm{D}$, where there can not be illumination effects such as shadows, brightness, reflections, etc. -see Appendix \#1.

\section{Communicability and Perception}

The main problem lies in the presentation of the cartographic information, which is increased by the size of the screens. Besides, in design it is not possible to resort to transparencies as a solution in the use of icons, river names, mountains, etc. The transparencies can double the visual information [13]. The use of transparent menus can occupy the whole screen or part of it. Studies carried out by Harrison et al. [14] demonstrate the efficacy of this technique in interfaces, since the transparencies focus the user's attention on the interface by highlighting the slide than its background. For a correct use of transparencies it is advisable to avoid backgrounds with a very transparent cartography, since they can prompt visual confusion if it is the case that either transparent menus or navigation keys are used.

Another way to duplicate the information in a screen is by using diffuse images. A set of graphic elements maintains its clearest colours and the rest, which have been diffused, acquire a greyer shade. The difference between a transparent image and a diffused one in an interface is that the first can be watched from the background of the screen, whereas the second cannot. In digital cartography there is more information in the interface when transparencies are used than when diffuse is used. Furthermore, through the use of the images one can curb the antinomy in the visual perception of maps. Any map is a display for a reader, for a user. From it, one must gather information and ideas. Often one must use a map as the basis for making a decision. This theme is found throughout the history of human activity, and the maps which exist at any particular time and in any particular culture mirror the environmental concerns, activities and behaviours of those whose created and used them. The communicability of the maps in digital supports must take into account two usability principles: easiness to remember and easiness to use [15]. 
As a rule, the users of hypermedia systems tend to use that kind of cartography where straight horizontal lines prevail, whether they are real or unreal. In the real case, perception is strengthened when the parallels are present. In the second case, the cartographer manages to bend the geographical territory in such a way that it matches the imagined horizontal line. This last action tends to cut down the effect of memory distortions. Systematic errors in perception and memory present a challenge to theories of perception and memory. Users perceived and remembered curves in mountains and rivers in maps as more symmetric than they actually were. Symmetry, useful for detecting and recognizing figures, lead to distortions in maps and graphs figures alike. Sometimes, users were asked to sketch the curves of the graphs or the rivers of the maps, and other times, they were asked questions about the content of the maps. This was done to induce a natural comprehension attitude toward the figures, and to prevent subjects from simply memorizing line.

We then asked judges who knew nothing about the hypotheses to rate whether the drawn curves and rivers were more or less symmetric than the original ones. The remembered curves, whether in maps or graphs, were judged more symmetric than the originals. These errors in the directions of symmetry, however, apparently occur in perception, not in memory [16]. When attention was directed to the symmetry of the curves, remembered curves were drawn more symmetric than when attention was drawn to the asymmetry of the curve. Modern cartograms in frequent use include automobile strip maps, the distorted map used by many rapid transit, i.e., underground or subway. These examples are often much easier to understand or use than their geographically correct counterparts.

The visual variables described by Jacques Bertin are very important [17], for example, size and value are efficient for portraying quantitative data, while form and colour are more suited for qualitative data. Textures and directions can be used for both types of data. In the absence of any conceptual or meaningful factors, there are often perceptual factors that provide a frame of reference. For maps, there is an additional conceptual factor that is typically perfectly linked with the perceptually salient axes, namely the cardinal directions, north-south and east-west. Thus far, the evidence for alignment has come either from maps and environments, where both perceptual and conceptual factors suggest the horizontal and vertical as a reference frame, or from visual blobs, where perceptual factors suggest the horizontal and vertical [16].

Another factor that must be taken into account in order to increase the user's perception and attention in the cartography, and to decrease the aporia is the distribution of the maps and the navigation zones in the interface. According to Leonardo Da Vinci, when he defines "La Divina Proporzione", that is, the proport-ional division of space in painting, since he sets down the areas that draw attention in a screen, there is a higher level of attention in the lower part of a rectangle than in its upper part. Besides, if two lines are drawn in the shape of a cross, you find that the right upper part has a higher perception and attention level than the lower one among Latin peoples [18].

\section{Antinomy and Aporia in Cartography}

In traditional paper cartography, the vector lines of the maps of a underground or/and subway are quickly assimilated by the population of a city. The colour, the letters and the numbers help the communicative process, reinforcing antinomy. Scales divides 
cartography in two big areas. The first one is the scale of 1:50.000 that uses the method called photogrametry, based on air frames and/or satellite images. In the case in which this scale becomes obsolete, there are other methods that are not considered in the present work because the aporia for the user of hypermedia systems is in the first group. In the classic structures of topography there's no problem, as the user has accustomed to bi-dimensional reading of cartographic information for many centuries.

The problem of aporia begins with digital urban cartography whose scale is of $1: 1000$, and in the symbology of thematic cartography above all. In urban cartography, the work in the real context is still necessary, i.e., go and measure the land in order to make a real analysis of the area, because from the sky it is difficult to define whether a path is practicable or not, whether a river is permanent or not, for example. In urban cartography the user can get his bearings quickly through the incorporation of cultural heritage, which must be included at the first level of visualization of the map and later on the kind of monument, building, etc. can be profiled. An excellent example is SimCity [12]. The tourist who visits a city for the first time and who, thanks to the 3D emulating maps, can get his bearings easily in the city. That is, that the emulation of the constructions in 3D favours antinomy.

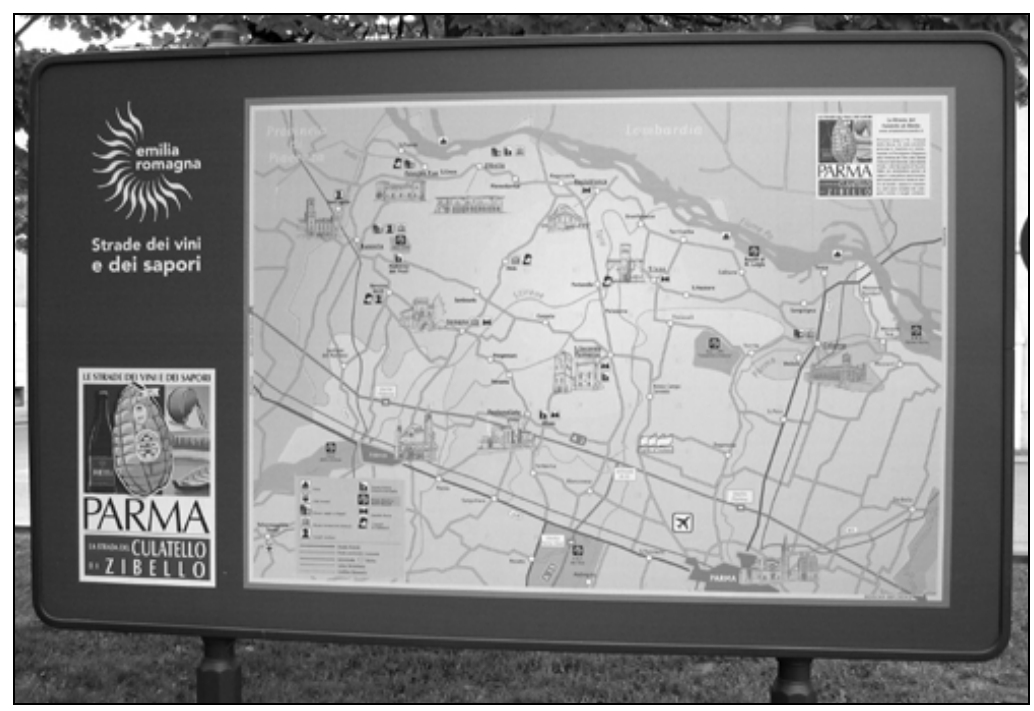

Fig. 1. In the poster, the $3 \mathrm{D}$ emulations of the monuments help to locate in a rural area where the main sites of interest are to be found

Previous and next posters -figures 1 and 2, of tourist maps from the Italian region of Emilia-Romagna. The first mentions a gastronomic zone to taste a typical product named "culatello" (ham). In the said map it can be seen how aporia can appear in thematic symbols, especially if you do not know the language of the country, since icons are coupled with texts in Italian. Furthermore, there is no such thing as a universal symbol of cultural heritage in thematic cartography. In these maps the aporia can also appear in different categories: population (evolution, structure, density, distribution, etc.); economy (usage of the ground, industrial and commercial activity, etc.); physical geography 
(climate, hydrographic, geology, etc.); society (education, work, justice, etc); equipment and services (sports, health facilities, tourism, communication and transport, etc). In the international design of interfaces there are different analyses or studies [19], [20], [21], but a database has not been established, with free access and which takes into consideration the different cultural factors. In the second poster -figure 2, there are two maps of some castles in the Emilia-Romagna region.

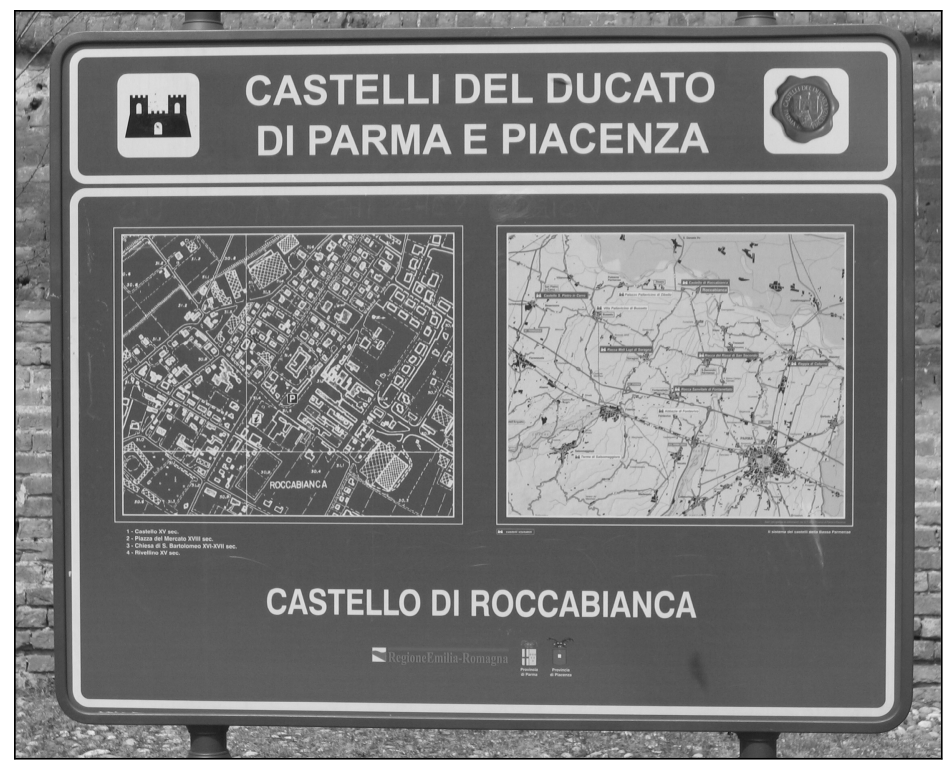

Fig. 2. In the left zone there is an aerial version of the zone (satellite photography) which does not indicate, for example, latitude or longitude. However, in the map both the parallel and meridian lines are marked.

If we observe the satellite image of the zone with the current contents on-line and the navigation elements available to the user, it is very hard for him to find orientation because the whole environment is a rural plain. As a result, the green colour prevails in the satellite image without additional indications. In the off-line interactive systems, In volo sull'Italia in $3 D$ [22], the use of transparent subtitles makes it possible to know in an efficient way the exact spot of the location seen from the satellite. Although the satellite images did not have the same quality as the current ones coming from NASA, as in the case of Google Maps, the interactive design was very good.

\section{Towards a Quality Design for Digital Cartography Online}

During the design of digital maps, the aporia can appear in the various stages:

1. Toponymy, i.e., the names of mountains, rivers, etc., that have to be written on the map. For example, in the Hispanic world the word Monaco refers to the Princedom of Monaco, whereas in Italian it means Monaco of Bavaria, that is to say, Munich. 
2. Geographical information: into the information derived from the analysis of the image in a map, we have to incorporate the administrative or political division. For example, the provincial limits, the traffic markings, etc.

3. The cartographic style, that relates to the elements constituting the map: tourist information -if it is a thematic map, colour and typography used for titles, etc.

The present maps with pictures from the satellite allow different ways of above vision: classic or 2D, 3D simulation and mixed -or a combination of the two previous ones. In most on-line maps, in the categories of 3D simulation or mixed, the user has no opportunity to adjust the vision angle. The cartographic image is mainly vectorial and allows the same actions as a pdf format document: enlarge and reduce the area, emphasize phrases with a labeller, insert comments under the picture, etc. The indications in the maps tend to globalize in the tourist field. Nevertheless, if the traffic markings are bad in a country, this high degree of aporia is also in its thematic maps. The user orientates himself very well, where the $2 \mathrm{D}$ format streets can be accompanied with a 3D simulation of the main buildings.

In the Google Maps -beta online version, the interactive system easily finds and shows the city of Vancouver, but when you want to have access to the capital of British Columbia, that is, the city of Victoria, there is no such city in the database. Automatically other cities of the world bearing the same name in other countries appear on the left margin of the screen -see figure 3 . However, the city of Victoria in Canada exists in the cartographic database of Google Maps.

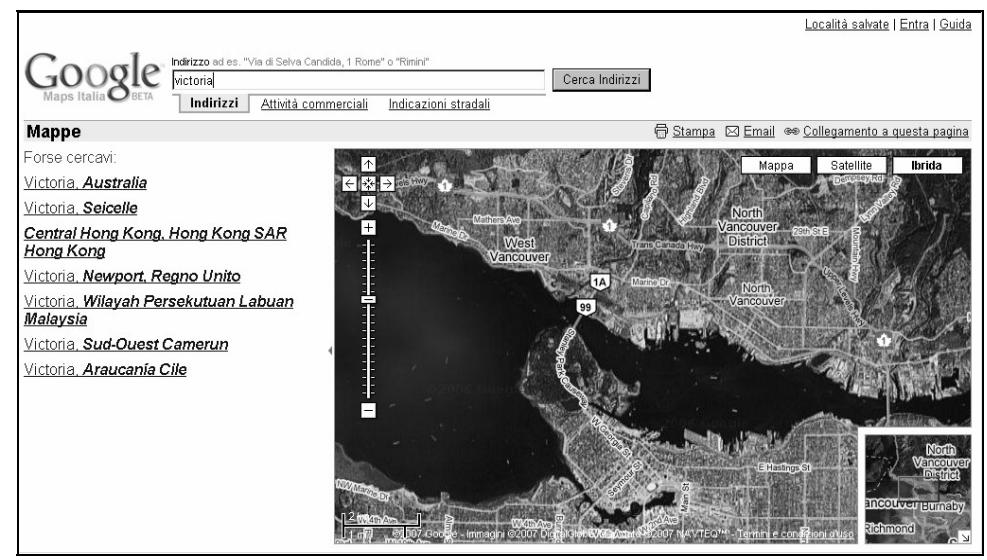

Fig. 3. The aporia appears in the accessibility of the information

An identical test has been carried out in the National Geographic on-line system and after visualizing the satellite map of Vancouver and searching for Victoria, the aforementioned city does show up quickly on the screen but in Hong Kong (China).

The use of the globe in the comic form is very positive from the standpoint of communicability, but its needs some changes to reduce aporia in the presentation of the information. For example, that globe with the shape of a comic should not generate shadows as in the Google Maps, and a semi-transparent version would be 


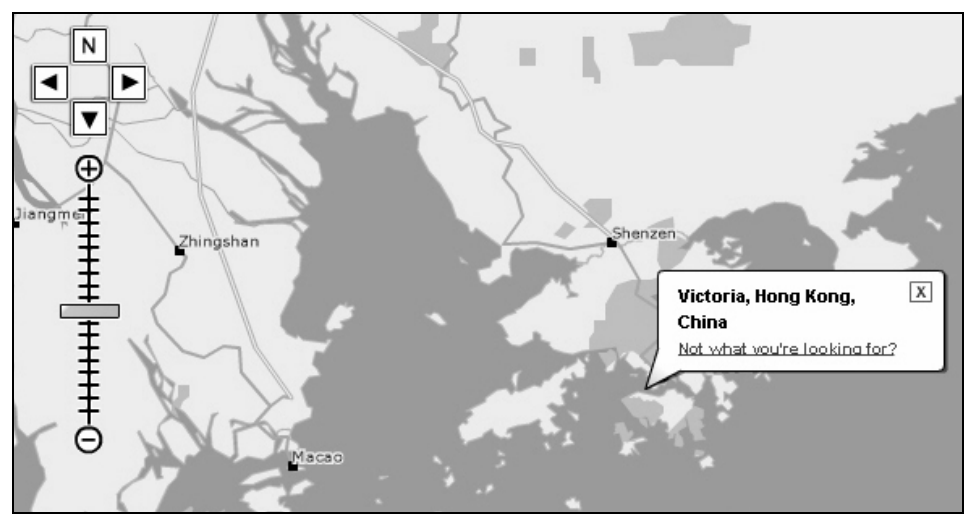

Fig. 4. A new search from the city of Vancouver to the city of Victoria, the globe in the comic form shape points at Hong Kong (http://plasma.nationalgeographic.com)

more convenient. In some cases, when the user chooses the mixed satellite option, that is, with the names of the main streets, roads, highways, etc., such a text is darkened by the shadow. The quality of the images in the maps slows down the presentation of the information in relation to the levels of approximation or distance from the zone of the map that is being watched at the moment, for instance, world, continent, country, city and street. In digital off-line cartography, the vectorial format prevails because of its high quality at the moment of approaching or distancing the perception of the map. In the fruition of maps via the Internet it is customary to resort to the bitmap format.

In some websites with satellite maps, the technique used to speed up the access to the map requested by the user is to divide the whole image into several parts. For example, it is the technique adopted by the National Geographic when it comes to presenting the map: it starts to generate the image in a partial manner from left to right. There is a loss of quality in the interaction of the user with the hypermedia system. However, from the standpoint of quality in the interactive communication it would be more convenient to use a transition effect of pixel fading which starts from a solid colour to the overall image of the map. The problems related to navigation in the system appear in the moment in which the user decides to locate a street that lies on the borders of the map. The system shifts the map automatically in order to locate it and point it out in the globe. Nevertheless, in the aforementioned shifting operation of the map, the original position gets lost and also the reference points, with which the user loses his bearings even more. The icons that mark the different points in the map do not allow the inference of the contents thereof. The user must select it and unfold it in order to see whether the contents are adjusted to what he is seeking. Moreover, if the listing happens to be extensive, the system inserts the pagination of the answer. As a consequence, this is hardly an ideal solution when the user does not know beforehand what he is trying to locate.

Perhaps it might work as a finder of addresses, phones, fax, etc. but in that case there are other databases on-line that do not insert a map for such activities. That is, to plan previously the information that must be shown, for example, to eliminate the option of watching non-existent highways in wide desert or rainforest regions, such as 
the Sahara or the Amazon. Leaving that space for another kind of theme, options such as can be the areas inhabited in those great regions (Sahara, i.e.). In the cartography of the cities, Anglo-Saxon users prefer maps with 3D perspectives to 2D maps. Latin European users prefer 2D maps, and Latin-American users remain indifferent in the face of a $2 \mathrm{D}$ or $3 \mathrm{D}$ map. The latter are also starting to value that cartography in the sightseeing areas they visit for the first time.

\section{Conclusions}

There is a fast diffusion of cartography in the new supports of multimedia information, however, a low quality in its fruition can be observed. Although the quality of cartographic pictures has increased in the last few years through the evolution of software, hardware procurement and image editing software of satellite data, etc. the design of interactive systems for this kind of material still remains superior in the hypermedia off-line systems compared with on-line ones. The main problem lies in the presentation of the information in the maps and the navigation zone in the screens of the different digital supports. On the one hand, there is a high amount of information in a tight space, on the other hand, there is no artificial or natural convention related to the iconography used for navigation. Accessibility in some cases is not only mistaken but also redundant. A way to solve this problem is to apply the criteria of richness of information according to the kind of user and the geographical context. The indications of the cultural heritage and the province, district limits etc., help orientation and location of what is being looked for in the satellite maps. However, the number of indications can either increase or decrease in relation to the zoom level the system offers to the user. In general terms, it is an option with several degrees of approximation and distancing. As a result, the user may get out of the map without being able to locate the information he is seeking, which exists in the database but can be found in a level that he/she has not visited. Another problem that fosters aporia in the interactive maps is that the pieces of information show in and off in relation to the zoom level, which is to some extent confusing to the inexpert user.

\section{Referentes}

1. Colapietro, V.: Semiotics. Paragon House, New York (1993)

2. Eco, U.: A Theory of Semiotics. Indiana University Press, Bloomington (1979)

3. Apple: Macintosh Human Interface Guidelines. Addison Wesley, Cupertino (1992)

4. Ben, C.: Notes from China: Handset Design. Interactions 13(4), 38-39 (2006)

5. Kjeldskov, J., Paay, J.: Public Pervasive Computing: Making the Invisible Visible. IEEE Computer 39(9), 60-65 (2006)

6. Davis, J., et al.: Simulations for Urban Planning: Designing for Human Values. IEEE Computer 39(9), 66-72 (2006)

7. Tate, A.: The Helpful Environment: Geographically Dispersed Intelligent Agents That Collaborate. IEEE Intelligent Systems 21(3), 57-61 (2006)

8. Cohen, P., McGee, D.: Tangible Multimodal Interfaces for Safety-Critical Applications. Communications of the ACM 47(1), 41-46 (2004) 
9. Robinson, et al.: Elements of Cartography. John Wiley and Sons, New York (1995)

10. Tufte, E.: The Visual Display of Quantitative Information. Graphics Press, Cheshire (2001)

11. Schnase, J., et al.: Semantic Database Modeling: Survey, Applications, and Research Issues. ACM Transactions on Information Systems 11(1), 27-50 (1993)

12. SimCity 4 CD-ROM: Electronics Arts, Redwood (2003)

13. Harrison, B., Vicente, K.: An Experimental Evaluation of Transparent Menu Usage. In: CHI'96, Vancouver, pp. 391-398. ACM Press, New York (1996)

14. Harrison, B., et al.: An Experimental Evaluation of Transparent User Interface Tools and Information Content. In: ACM Symposium on User Interface Software and Technology (UIST '95), Pittsburgh, pp. 99-106. ACM Press, New York (1995)

15. Nielsen, J.: The Usability Engineering Life Cycle. IEEE Computer 25(3), 12-22 (1992)

16. Kaiser, M., Ellis, S.: Pictorial Communication in Virtual and Real Environments. Taylor \& Francis, Washington (1993)

17. Bertin, J.: Sémiologie graphique: Les diagrammes, les réseaux, les cartes. EHESS, Paris (2005)

18. Cipolla-Ficarra, F.: Evaluation and Communication Techniques. In: Multimedia Product Design for on the Net University Education. Multimedia on the Net, pp. 151-165. Springer-Verlag, Heidelberg (1996)

19. Kellog, W., Thomas, J.: Cross-Cultural Perspective on Human-Computer Interaction. SIGCHI 25(2), 40-45 (1993)

20. Nielsen, J., del Galdo, E.: International User Interfaces. Wiley, New York (1996)

21. Shneiderman, B.: Designing the User Interface. Addison-Wesley, New York (1997)

22. In volo sull'Italia in 3D CD-ROM: Infmedia, Rome (2003)

\section{Appendix 1: Quality Metric Antinomy}

Table 1. Element, components/attributes and design categories: Content (CO), Dynamic (DY), Panchronic (PA), Presentation (PR) and Structure (ST)

\begin{tabular}{lll}
\hline Element & Components and/or attributes & Design categories \\
\hline 1) Bird Vision & Control of fruition and orientation & DY, PA, PR, ST \\
\hline $\begin{array}{l}\text { 2) Geographical } \\
\text { Information }\end{array}$ & Sense of direction and transparence & CO, PR \\
\hline 3) Perception and & $\begin{array}{l}\text { Maps structure, topology on the screen, PR } \\
\text { Memory }\end{array}$ & $\begin{array}{l}\text { inference and 3D constructions } \\
\text { melation between primary and secondary CO, PR }\end{array}$ \\
\hline 4) Toponymy & $\begin{array}{l}\text { meaning: univocal, biunivocal, and semiosis } \\
\text { iconography Languages and international }\end{array}$ & \\
& Realism, emulation, simulation, and richness & CO, DY, PA, PR, \\
& & ST \\
\hline 5) Cartographic \\
Style
\end{tabular}

